\title{
Abeo-taxanes from Taxus mairei
}

\author{
Shung-Jim Yang, Jim-Min Fang*, Yu-Shia Cheng \\ Department of Chemistry, National Taiwan University, Taipei 106, Taiwan, R.O.C. \\ Revised 9 March 1998
}

\begin{abstract}
One new taxane (baccatin III 13-cinnamate), two new $11(15 \rightarrow 1)$ abeo-taxanes (taxumains $\mathrm{A}$ and $\mathrm{B}$ ) and 15 known taxanes were isolated from the twigs of Taxus mairei. The structures of new compounds were determined by spectroscopic methods. (C) 1998 Published by Elsevier Science Ltd. All rights reserved.
\end{abstract}

Keywords: Taxus mairei; Taxanes; Taxus; Twigs; Taxanes; 11(15 $\rightarrow$ 1)abeo-taxanes

\section{Introduction}

The chemical constituents of the plants of the Taxus genus have been extensively investigated (Appendino, 1995; Kingston, Molinero \& Rimoldi, 1993; Chen, 1990), partly due to the discovery of the antitumor agent, taxol (Wani et al., 1971). T. mairei is the only species belonging to the genus Taxus found in Taiwan. A few taxane derivatives have been isolated from the heartwood of this plant (Yeh, Wang \& Chen, 1988; Min, Jiang \& Liang, 1989). In a previous paper (Yang, Fang \& Cheng, 1996), we have reported 16 taxane diterpenes having $\mathrm{C} 4(20)$-exocyclic double bonds isolated from the twigs. As the continuation of our chemical investigation on $T$. mairei, we report herein 18 additional taxanes, including two new $11(15 \rightarrow 1)$ abeo-taxanes.

\section{Results and discussion}

A concentrated acetone extract of the twigs of $T$. maire $i$ was taken in ethyl acetate, the soluble part was concentrated and chromatographed to give 18 taxanes. By analysis of their physical and spectral properties

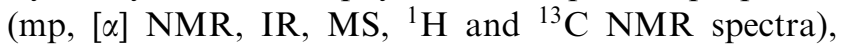
15 known compounds were identified as baccatin III 1 (Henilh et al., 1984), 13-dehydro-10-deacetylbaccatin

\footnotetext{
* Author to whom correspondence should be sent.
}

III 2 (Barboni et al., 1994), 13-dehydrobaccatin III 3 (Wani et al., 1971), taxol 5 (Wani et al., 1971), taxol C 6(Kitagawa et al., 1995), taxol 7-( $\beta$-triacetylxyloside) 7 (Henilh et al., 1984), 1-deoxybaccatin IV 8 (Della Casa de Marcano \& Halsall, 1975), baccatin IV 9 (Della Casa de Marcano \& Halsall, 1975), 1-deoxybaccatin VI 10 (Min, Jiang \& Liang, 1989), baccatin VI 11 (Min, Jiang \& Liang, 1989), 2 $\alpha$-benzoyloxy-4 $\alpha, 20$-dihydroxy- $5 \alpha, 7 \beta, 9 \alpha, 10 \beta, 13 \alpha$-pentaacetoxytax-11-ene 12 (Liang \& Kingston, 1993), $2 \alpha$-benzoyloxy- $4 \alpha, 5 \alpha$-dihydroxy-7 $\beta, 9 \alpha, 10 \beta, 13 \alpha, 20$-pentaacetoxytax-11-ene 13 (Liang \& Kingston, 1993), 4 $\beta, 20$-epoxy- $2 \alpha, 5 \alpha, 10 \beta, 13 \alpha-$ tetraacetoxy- $1 \beta, 7 \beta, 9 \alpha$-trihydroxytax-11-ene 14 (Shen, Tai \& Chen, 1996), 1 $\alpha, 7 \beta$-dihydroxy-4 $\beta, 20$-epoxy$2 \alpha, 5 \alpha, 9 \alpha, 10 \beta, 13 \alpha$-pentaacetoxytax-11-ene 15 (Chu et al., 1993), and $1 \beta$-hydroxybaccatin I 16 (Yeh, Wang \& Chen, 1988).

The new taxane 4 was determined to be baccatin III 13-cinnamate by analysis of its ${ }^{1} \mathrm{H}$ and ${ }^{13} \mathrm{C}$ NMR spectra. The assignments of individual protons and carbons were made by the assistance of ${ }^{1} \mathrm{H}-{ }^{1} \mathrm{H}$ COSY, HMQC and HMBC spectra. The carbon resonance at $\delta_{\mathrm{C}} 166.2$ was attributable to the cinnamoyl group, of which $E$-configuration was inferred from a large coupling constant of $16 \mathrm{~Hz}$ between the two olefinic protons at $\delta 7.83$ and 6.51. The HMBC spectrum showed the correlation of cinnamoyl carbonyl (at $\delta_{\mathrm{C}} 166.2$ ) with the $\mathrm{C}-13$ proton (at $\delta_{\mathrm{H}} 6.18$ ), and the correlation of benzoyl carbonyl (at $\delta_{\mathrm{C}} 166.9$ ) with the $\mathrm{C}-2$ proton (at $\delta_{\mathrm{H}}$ 5.66). The characteristic resonances of oxetane occurred at $\delta_{\mathrm{H}} 4.15$ and 4.31 (AB type, $J=8.4 \mathrm{~Hz}$ ). 


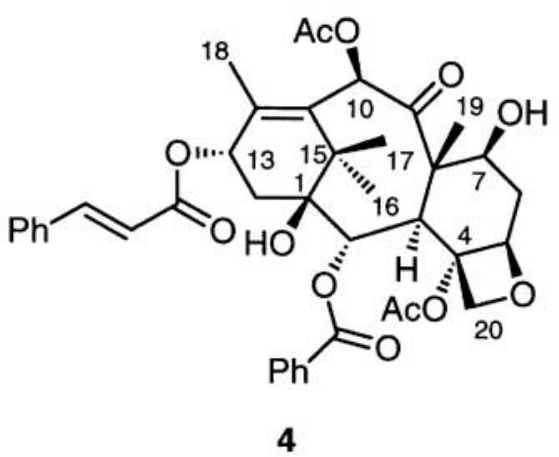

The $10 \alpha-\mathrm{H}$ occurring at $\delta 6.32$ (s) was consistent with the structure having C-10 acetoxy and C-9 carbonyl groups. The H-3 occurred at a relatively low field of $\delta$ 3.85 , presumably due to the deshielding effect of the carbonyl group at C-9.

Compound $\mathbf{1 7}$ (taxumain A) and $\mathbf{1 8}$ (taxumain B) are $11(15 \rightarrow 1)$ abeo-taxanes. The molecular formula of 17 was deduced to be $\mathrm{C}_{30} \mathrm{H}_{44} \mathrm{O}_{13}$ as its molecular ion occurred at $m / z 612.2722$ in the HR mass spectrum. From the NMR spectral analysis, no oxetane group was present in 17; instead, a carbon resonance at $\delta 62.7$ and the proton resonances at $\delta 3.75(d$, $J=11.6 \mathrm{~Hz})$ and $4.19(d d, J=11.6,8.4 \mathrm{~Hz})$ indicated the presence of a $\mathrm{CH}_{2} \mathrm{OAc}$ moiety. The assignment of $\mathrm{H}-3$ at $\delta 2.73(d d, J=8.0,4.4 \mathrm{~Hz})$ was supported by its correlation with H-4 (at $\delta$ 2.12) in the ${ }^{1} \mathrm{H}-{ }^{1} \mathrm{H}$ COSY spectrum. Similarly, $\mathrm{H}-2$ at $\delta 5.69$ $(d, \quad J=8.0 \mathrm{~Hz})$ was assigned. The presence of a hydroxy substituent on C-15 was indicated by the carbon resonance at $\delta$ 76.3. The HMBC spectrum also showed the correlations of $\mathrm{H}-2$ with $\mathrm{C}-1$ (at $\delta$ 67.6), C-4 (at $\delta 41.5$ ) and C-15, in agreement with the structure of $11(15 \rightarrow 1)$ abeo-taxane 17 . The large coupling constant $J_{9,10}=10.4 \mathrm{~Hz}$ was consistent with the trans relationship of H-9 (at $\delta$ 5.53) and H-10 (at $\delta$ 4.54). The NOESY experiment showed correlation of $\mathrm{H}-2$ (at $\delta$ 5.69) with $\mathrm{H}-9$ and $\mathrm{H}-19$ (at $\delta 0.98$ ), supporting the assigned stereochemistry.

Compound 18 had ${ }^{1} \mathrm{H}$ and ${ }^{13} \mathrm{C}$ NMR spectral characteristics similar to those of $\mathbf{1 7}$, except that the $\mathrm{H}-13$ geminal to an acetoxy group appeared at a lower field of $\delta$ 5.57. The exact mass of molecular ion at $\mathrm{m} / \mathrm{z} 654.2813$ led to a molecular formula $\mathrm{C}_{32} \mathrm{H}_{46} \mathrm{O}_{14}$. Thus compound $\mathbf{1 8}$ was determined to be the 13-acetyl derivative of 17. By the assistance of HMQC and HMBC spectra, individual protons and carbons were assigned. The signals of $\mathrm{H}-9$ and $\mathrm{H}-10$ occurred at $\delta 5.59(d, J=10.2 \mathrm{~Hz})$ and $4.55(d$, $J=10.2 \mathrm{~Hz}$ ), respectively. The correlation of C-13 (at $\delta_{\mathrm{C}} 78.7$ ) with one of $\mathrm{H}-14$ (at $\delta_{\mathrm{H}} 1.67$ ) was shown in the HMBC spectrum. In agreement with this assign-

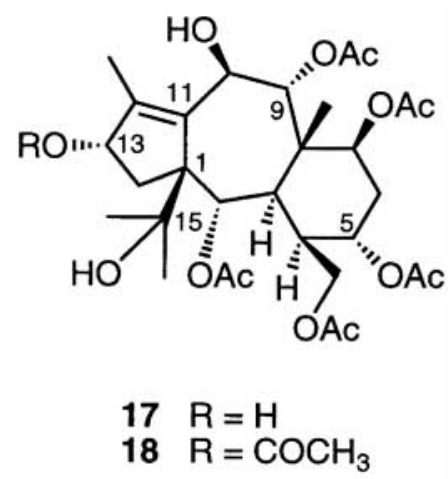

ment, $\mathrm{H}-13$ appeared at a low field of $\delta 5.57(b r t)$ due to the inductive effect of an acetoxy substituent at $\mathrm{C}-13$.

In summary, the twigs of $T$. mairei contain various taxane diterpenes. The known abeo-taxanoids usually have either 4-oxy substituents or 4(20)-double bonds (Kingston, Samaranayake \& Ivey, 1990; Zhang et al., 1994; Fuji et al., 1995). Taxumain A and taxumain B are, however, the first reported abeo-taxanes lacking any oxygenated functionality at C-4.

\section{Experimental}

\subsection{General}

Yanagimoto (or MP-500D) micro melting point apparatus; Jasco Dip-180 digital polarimeter, Finnigan TSQ-46c mass spectrometer; ${ }^{1} \mathrm{H}$ NMR: 300 or $400 \mathrm{MHz} ;{ }^{13} \mathrm{C}$ NMR: 75 or $100 \mathrm{MHz}$; Waters M-45 high-pressure liquid chromatograph with Hibar Lichrosorb Si 60 column $(10 \mu \mathrm{m}$ or $7 \mu \mathrm{m}, 25 \times 1 \mathrm{~cm}$ i.d.) were used.

\subsection{Plant material}

The twigs $(1.2 \mathrm{~kg})$ of $T$. mairei were collected in the remote mountains at an elevation of $c a .2100 \mathrm{~m}$ (Tong-Shi, Taichung county). A voucher specimen is deposited in the Herbarium of National Taiwan University. The air-dried material was exhaustively extracted with acetone $(7 \mathrm{~L} \times 3)$. The acetone extract was concentrated to give $100 \mathrm{~g}$ of residue, which was diluted with water and extracted with EtOAc $(1 \mathrm{~L} \times 3)$. The combined EtOAc extracts were concentrated to give an oil $(75 \mathrm{~g})$, which was absorbed with $110 \mathrm{~g}$ of $\mathrm{SiO}_{2}$ and then chromatographed on a column packed with $650 \mathrm{~g}$ of $\mathrm{SiO}_{2}$ by elution with gradients of hexane, EtOAc and acetone. The portion obtained from the elution of EtOAc/hexane (70-90\%) was further subjected to flash chromatography and HPLC 
with elution of EtOAc/ $\mathrm{CH}_{2} \mathrm{Cl}_{2}(50-60 \%)$ or acetone/ hexane (20-40\%). Compounds $8(20 \mathrm{mg}), 10(22 \mathrm{mg})$, $9(25 \mathrm{mg}), \mathbf{1 1}(17 \mathrm{mg}), \mathbf{1 6}(30 \mathrm{mg}), \mathbf{1 5}(8 \mathrm{mg}), \mathbf{3}$ (12 mg), 4 (8 mg), 1 (25 mg), 13 (7 mg), $12(5 \mathrm{mg}), \mathbf{2}$ $(20 \mathrm{mg}), \mathbf{1 4}(16 \mathrm{mg}), \mathbf{1 8}(14 \mathrm{mg}), \mathbf{1 7}(22 \mathrm{mg}), \mathbf{6}(6 \mathrm{mg})$, $5(10 \mathrm{mg})$ and $7(7 \mathrm{mg})$ were obtained in the ascending order of polarity.

\subsection{Baccatin III (1)}

Needles, mp 238-240,$[\alpha]_{\mathrm{D}}^{29}-50\left(\mathrm{CHCl}_{3} ;\right.$ c 1.0) \{ [8], mp 236-238 , $[\alpha]_{\mathrm{D}}-54\left(\mathrm{CHCl}_{3} ;\right.$ c 1.2$\left.)\right\}$.

\subsection{3-Dehydro-10-deacetylbaccatin III (2)}

Needles, mp $167-170^{\circ},[\alpha]_{\mathrm{D}}^{23}-12.4\left(\mathrm{CHCl}_{3} ; c\right.$ 1.9) $\left\{[9] \mathrm{mp} 168-169^{\circ}\right\}$.

\subsection{3-Dehydrobaccatin III (3)}

Needles, mp $211-213^{\circ},[\alpha]_{\mathrm{D}}^{29}-32.4\left(\mathrm{CHCl}_{3} ; c\right.$ 1.2) $\left\{[4], \mathrm{mp} 210-212^{\circ},[\alpha]_{\mathrm{D}}-40\left(\mathrm{CHCl}_{3} ; c\right.\right.$ 1.8)\}.

\subsection{Baccatin III 13-cinnamate (4)}

Gum, $[\alpha]_{\mathrm{D}}^{25}-75.7 \quad\left(\mathrm{CHCl}_{3} ; c \quad 1.0\right)$. IR $v_{\max }^{\mathrm{KBr}} \mathrm{cm}^{-1}$ : $3428,1712,1596,1509,1176 .{ }^{1} \mathrm{H}$ NMR $\left(\mathrm{CDCl}_{3}\right.$, $400 \mathrm{MHz}): \delta 1.11(3 \mathrm{H}, s, \mathrm{H}-17), 1.22(3 \mathrm{H}, s, \mathrm{H}-16)$, $1.66(3 \mathrm{H}, s, \mathrm{H}-19), 2.00(3 \mathrm{H}, s, \mathrm{H}-18), 2.22(1 \mathrm{H}, d d$, 8.4, 15.2 Hz, H-14), $2.23(3 \mathrm{H}, s, \mathrm{AcO}), 2.26(3 \mathrm{H}, s$, AcO), $2.41(1 \mathrm{H}, d d, 8.4,15.2 \mathrm{~Hz}, \mathrm{H}-14), 2.55(2 \mathrm{H}, m$, $\mathrm{H}-6), 3.85(1 \mathrm{H}, d, 6.8 \mathrm{~Hz}, \mathrm{H}-3), 4.31(1 \mathrm{H}, d, 8.4 \mathrm{~Hz}$, $\mathrm{H}-20), 4.15(1 \mathrm{H}, d, 8.4 \mathrm{~Hz}, \mathrm{H}-20), 4.45(1 \mathrm{H}, m, \mathrm{H}-7)$, $4.96(1 \mathrm{H}, d, 8.1 \mathrm{~Hz}, \mathrm{H}-5), 5.66(1 \mathrm{H}, d, 6.8 \mathrm{~Hz}, \mathrm{H}-2)$, $6.18(1 \mathrm{H}, t, 8.4 \mathrm{~Hz}, \mathrm{H}-13), 6.32(1 \mathrm{H}, s, \mathrm{H}-10), 6.51(1$ $\mathrm{H}, d, 16.0 \mathrm{~Hz}), 7.43(3 \mathrm{H}, m), 7.46(2 \mathrm{H}, m), 7.56(2 \mathrm{H}$, $m), 7.57(1 \mathrm{H}, m), 7.83(1 \mathrm{H}, d, 16.0 \mathrm{~Hz}), 8.04(2 \mathrm{H}, d$, $7.2 \mathrm{~Hz}) .{ }^{13} \mathrm{C}$ NMR $\left(\mathrm{CDCl}_{3}, 100 \mathrm{MHz}\right): \delta 9.4(\mathrm{C}-19)$, 15.5 (C-18), 20.9 (AcO), 21.3 (C-16), 22.6 (AcO), 26.8 (C-17), 35.6 (C-14), 36.2 (C-6), 43.0 (C-15), 45.8 (C-3), 58.7 (C-8), 70.0 (C-13), 72.3 (C-7), 74.8 (C-2), 75.7 (C10), 76.4 (C-20), 79.2 (C-1), 81.0 (C-4), 84.3 (C-5), 116.7 (cinn.), 128.2, 128.6, 129.0, 129.1, 130.0, 130.9 (2 C, C-11 and $\mathrm{BzO}), 133.7,133.8,143.1$ (C-12), 146.7 (cinn.), 166.2 (cinn.), 166.9 (BzO), 169.8 (AcO), 171.3 (AcO), 203.8 (C-9). FAB-MS (NBA) $m / z$ (rel. int.): 657 [M-OAc] $^{+}$(5), 639 (7), 579 (3), 509 (4), 327 (10), 289 (20), 154 (70), 131 (100). HR-MS for $\mathrm{C}_{38} \mathrm{H}_{41} \mathrm{O}_{10}$ [M-OAc] $^{+}$requires: 657.2700. Found: 657.2704.

\subsection{Taxol (5)}

Needles, mp 195-200,$[\alpha]_{\mathrm{D}}^{29}-30\left(\mathrm{CHCl}_{3} ;\right.$ c 0.9$)\{[4]$, mp $198-203^{\circ},[\alpha]_{\mathrm{D}}-42\left(\mathrm{CHCl}_{3} ;\right.$ c 1.0$\left.)\right\}$.

\subsection{Taxol C (6)}

Needles, mp 200-204,$[\alpha]_{\mathrm{D}}^{29}-50\left(\mathrm{CHCl}_{3} ; c c c 0.5\right)$ $\left\{[10], \mathrm{mp} 202-204^{\circ},[\alpha]_{\mathrm{D}}-62\left(\mathrm{CHCl}_{3} ; c 0.8\right)\right\}$.

\subsection{Taxol 7-( $\beta$-triacetylxyloside) (7)}

Gum, $[\alpha]_{\mathrm{D}}^{29}-10\left(\mathrm{CHCl}_{3} ; c c 0.8\right)\left\{[8], \quad[\alpha]_{\mathrm{D}}-23\right.$ $\left.\left(\mathrm{CHCl}_{3} ; c 1.0\right)\right\}$.

\subsection{1-Deoxybaccatin IV (8)}

Needles, mp 259-262,$[\alpha]_{\mathrm{D}}^{29}+88\left(\mathrm{CHCl}_{3} ; c\right.$ 1.2) $\left\{[11], \mathrm{mp} 259-260^{\circ},[\alpha]_{\mathrm{D}}+99\left(\mathrm{CHCl}_{3} ; c\right.\right.$ 1.5)\}.

\subsection{Baccatin IV (9)}

Needles, mp $253-255^{\circ},[\alpha]_{\mathrm{D}}^{29}+15\left(\mathrm{CHCl}_{3} ; c\right.$ 1.6) $\left\{[11], \mathrm{mp} 254-255^{\circ},[\alpha]_{\mathrm{D}}+19\left(\mathrm{CHCl}_{3} ; c 1.0\right)\right\}$.

\subsection{1-Deoxybaccatin VI (10)}

Needles, mp 219-222 ${ }^{\circ},[\alpha]_{\mathrm{D}}^{29}-18\left(\mathrm{CHCl}_{3} ; c\right.$ 1.2) \{[6], mp $\left.220-221^{\circ},[\alpha]_{\mathrm{D}}-21.2\left(\mathrm{CHCl}_{3} ; c 1.0\right)\right\}$.

\subsection{Baccatin VI (11)}

Needles, mp $238-241^{\circ},[\alpha]_{\mathrm{D}}^{29}-15\left(\mathrm{CHCl}_{3} ; c\right.$ 1.6) $\{[6]$, mp 239-241 $\left.{ }^{\circ},[\alpha]_{\mathrm{D}}-9\left(\mathrm{CHCl}_{3} ; c 1.9\right)\right\}$.

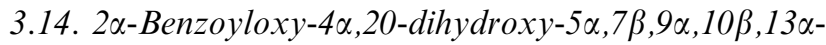
pentaacetoxytax-11-ene (12)

Needles, mp $112-115^{\circ},[\alpha]_{\mathrm{D}}^{29}-18\left(\mathrm{CHCl}_{3} ; c c c .5\right)$ $\left\{[12], \mathrm{mp} 114-115^{\circ},[\alpha]_{\mathrm{D}}-30\left(\mathrm{CHCl}_{3} ; c 0.1\right)\right\}$.

\subsection{5. $2 \alpha$-Benzoyloxy- $4 \alpha, 5 \alpha$-dihydroxy- $7 \beta, 9 \alpha, 10 \beta, 13 \alpha, 20$ -} pentaacetoxytax-11-ene (13)

Needles, mp $120-124^{\circ},[\alpha]_{\mathrm{D}}^{29}-20\left(\mathrm{CHCl}_{3} ; c c 0.4\right)$ $\left\{[12], \mathrm{mp} 122-123^{\circ},[\alpha]_{\mathrm{D}}-37\left(\mathrm{CHCl}_{3} ; c 0.1\right)\right\}$.

3.16. $4 \beta, 20$-Epoxy- $2 \alpha, 5 \alpha, 10 \beta, 13 \alpha$-tetraacetoxy- $1 \beta, 7 \beta, 9 \alpha-$ trihydroxytax-11-ene (14)

Amorphous powder, $[\alpha]_{\mathrm{D}}^{29}+43\left(\mathrm{CHCl}_{3} ; c 0.8\right)\{[13]$, $\left.[\alpha]_{\mathrm{D}}+46\left(\mathrm{CHCl}_{3} ; c 1.5\right)\right\}$.

3.17. $1 \alpha, 7 \beta$-Dihydroxy- $4 \beta, 20$-epoxy- $2 \alpha, 5 \alpha, 9 \alpha, 10 \beta, 13 \alpha-$ pentaacetoxytax-11-ene (15)

Amorphous powder, $[\alpha]_{\mathrm{D}}^{29}+120 \quad\left(\mathrm{CHCl}_{3} ; \quad c \quad 0.6\right)$ $\left\{[14],[\alpha]_{\mathrm{D}}+138.7\left(\mathrm{CHCl}_{3} ; c 1.2\right)\right\}$. 


\subsection{1ß-Hydroxybaccatin I (16)}

Needles, mp 260-262,$[\alpha]_{\mathrm{D}}^{29}+65\left(\mathrm{CHCl}_{3} ; c\right.$ 1.4) $\{[5]$, mp 260-261,$\left.[\alpha]_{\mathrm{D}}+71.8\left(\mathrm{CHCl}_{3} ; c 1.0\right)\right\}$.

\subsection{Taxumain $A(\mathbf{1 7})$}

Needles, mp $284-286^{\circ} \mathrm{C},[\alpha]_{\mathrm{D}}^{23}-11.1\left(\mathrm{CHCl}_{3} ; c\right.$ 1.5). IR $v_{\max }^{\mathrm{KBr}} \mathrm{cm}^{-1}: 3414,1736,1028 .{ }^{1} \mathrm{H} \mathrm{NMR}\left(\mathrm{CDCl}_{3}\right.$, $400 \mathrm{MHz}): \delta 0.98$ (3 H, s, H-19), 1.02 (3 H, s, H-17), 1.20 (3 H, s, H-16), 1.69 (1 H, dd, 7.2, 14,4 Hz, H-14), 1.82 (2 H, m, H-6), 1.84 (3 H, $s, \mathrm{H}-18), 2.02$ (3 H, $s$, AcO), 2.03 (3 H, $s, \mathrm{AcO}), 2.05$ (3 H, s, AcO), 2.06 (3 $\mathrm{H}, s, \mathrm{AcO}), 2.08(3 \mathrm{H}, s, \mathrm{AcO}), 2.10$ (1 H, m, H-14), $2.73(1 \mathrm{H}, d d, 4.4,8.0 \mathrm{~Hz}, \mathrm{H}-3), 2.12$ (1 H, m, H-4), $3.75(1 \mathrm{H}, d, 11.6 \mathrm{~Hz}, \mathrm{H}-20), 4.19(1 \mathrm{H}, d d, 8.4$, $11.6 \mathrm{~Hz}, \mathrm{H}-20), 4.95$ (1 H, br s, H-5), 4.54 (1 H, d, $10.4 \mathrm{~Hz}, \mathrm{H}-10), 4.57$ (1 H, br t, H-13), 5.22 (1 H, dd, 5.2, $11.2 \mathrm{~Hz}, \mathrm{H}-7), 5.53$ (1 H, $d, 10.4 \mathrm{~Hz}, \mathrm{H}-9), 5.69$ (1 $\mathrm{H}, d, 8.0 \mathrm{~Hz}, \mathrm{H}-2) .{ }^{13} \mathrm{C} \mathrm{NMR}\left(\mathrm{CDCl}_{3}, 100 \mathrm{MHz}\right): \delta$ 11.0 (C-18), 14.1 (C-19), 20.9, 21.1, 21.2, 21.3, 21.4, 25.7 (C-16), 27.7 (C-17), 29.1 (C-6), 40.5 (C-14), 40.6 (C-3), 41.5 (C-4), 43.1 (C-8), 62.7 (C-20), 66.7 (C-10), 67.6 (C-1), 68.0 (C-2), 68.8 (C-7), 70.4 (C-5), 76.3 (C15), 77.0 (C-13), 79.0 (C-9), 138.2 (C-11), 145.7 (C-12), 168.8, 170.7, 170.8, 171.1, 171.2. FAB-MS (NBA) $\mathrm{m} / z$ (rel. int.): $635[\mathrm{M}+\mathrm{Na}]^{+}$(8), 594 (12), 577 (18), 535 (10), 417 (30), 307 (20), 154 (100). HR-MS for $\mathrm{C}_{30} \mathrm{H}_{44} \mathrm{O}_{13}$ requires: 612.2782. Found: 612.2722.

\subsection{Taxumain B (18)}

Gum, $[\alpha]_{\mathrm{D}}^{25}-15.2\left(\mathrm{CHCl}_{3} ; c c 0.9\right)$. IR $v_{\max }^{\mathrm{K} \mathrm{Br}} \mathrm{cm}^{-1}$ : 3414, 1732, 1025. ${ }^{1} \mathrm{H}$ NMR $\left(\mathrm{CDCl}_{3}, 300 \mathrm{MHz}\right): \delta 1.01$ (3 H, s, H-19), 1.10 (3 H, s, H-17), 1.22 (3 H, s, H-16), 1.67 (1 H, dd, 7.5, 14.4 Hz, H-14), 1.85 (2 H, m, H-6), 1.87 (3 H, s, H-18), 2.01 (3 H, s, AcO), 2.02 (3 H, $s$, AcO), 2.03 (3 H, $s, \mathrm{AcO}), 2.04$ (3 H, s, AcO), 2.08 (3 $\mathrm{H}, s, \mathrm{AcO}), 2.09(3 \mathrm{H}, s, \mathrm{AcO}), 2.11(1 \mathrm{H}, m, \mathrm{H}-14)$, 2.13 (1 H, m, H-4), $2.66(1 \mathrm{H}, d d, J=4.2,8.1 \mathrm{~Hz}, \mathrm{H}-$ 3), $3.77(1 \mathrm{H}, d, J=12.0 \mathrm{~Hz}, \mathrm{H}-20), 4.19(1 \mathrm{H}, d d$, $8.4, J=12.0 \mathrm{~Hz}, \mathrm{H}-20), 4.55(1 \mathrm{H}, d, J=10.2 \mathrm{~Hz}, \mathrm{H}-$ 10), 4.97 (1 H, br s, H-5), 5.23 (1 H, dd, $J=5.7$, $10.8 \mathrm{~Hz}, \mathrm{H}-7), 5.59$ (1 H, $d, J=10.2 \mathrm{~Hz}, \mathrm{H}-9), 5.57$ (1
$\mathrm{H}$, br $t, J=7.5 \mathrm{~Hz}, \mathrm{H}-13), 5.71(1 \mathrm{H}, d, J=8.1 \mathrm{~Hz}, \mathrm{H}-$ 2). ${ }^{13} \mathrm{C} \mathrm{NMR}\left(\mathrm{CDCl}_{3}, 75 \mathrm{MHz}\right): \delta 11.0$ (C-18), 14.2 (C-19), 20.8, 21.0, 21.1, 21.2, 21.3, 21.4, 25.7 (C-16), 27.8 (C-17), 29.0 (C-6), 38.1 (C-14), 40.6 (C-3), 41.5 (C-4), 43.0 (C-8), 62.7 (C-20), 66.4 (C-10), 67.7 (C-1), 67.9 (C-7), 68.7 (C-2), 70.5 (C-5), 76.4 (C-15), 78.7 (C13), 79.7 (C-9), 140.5 (C-11), 142.1 (C-12), 168.5, 168.7, 170.6, 170.9, 171.1 (2 C). FAB-MS (NBA) $\mathrm{m} / z$ (rel. int.): $677[\mathrm{M}+\mathrm{Na}]^{+}(10), 637(20), 577(30), 517$ (22), 475 (23), 417 (25), 136 (100). HR-MS for $\mathrm{C}_{32} \mathrm{H}_{46} \mathrm{O}_{14}$ requires: 654.2888. Found: 654.2813.

\section{References}

Appendino, G. (1995). Natural Product Reports, 12, 349.

Barboni, L., Gariboldi, P., Torregiani, E., Appendino, G., Gabetta, B., \& Bombardelli, E. (1994). Phytochemistry, 36, 987.

Chen, W.-M. (1990). Acta Pharmacy Sinica, 25, 227.

Chu, A., Zajicek, J., Davin, L. B., Lewis, N. G., \& Croteau, R. (1993). Phytochemistry, 34, 473.

Della Casa de Marcano, D. P., \& Halsall, T. G. (1975). Journal of Chemical Society, Chemical Communications, 365.

Fuji, K., Tanaka, K., Li, B., Shingu, T., Yokoi, T., Sun, H., \& Taga, T. (1995). Tetrahedron, 51, 10175.

Henilh, V., Blechert, S., Colin, M., Guenard, D., Picot, F., Potier, P., \& Varenne, P. (1984). Journal of Natural Products, 47, 131.

Kingston, D. G. I., Molinero, A. A., \& Rimoldi, J. M. (1993). Progress in the Chemistry of Organic Natural Products. Wien: SpringerVerlag, 61, p. 1.

Kingston, D. G. I., Samaranayake, G., \& Ivey, C. A. (1990). Journal of Natural Products, 53, 1.

Kitagawa, I., Mahmud, T., Kobayashi, M., Roemanty, O., \& Shibuya, H. (1995). Chemical and Pharmaceutical Bulletin, 43, 365.

Liang, J., \& Kingston, D. G. I. (1993). Journal of Natural Products, 56, 594.

Min, Z.-D., Jiang, H., \& Liang, J. Y. (1989). Acta Pharmacy Sinica, 24, 673.

Shen, Y.-C., Tai, H.-R., \& Chen, C.-Y. (1996). Journal of Natural Products, 59, 173.

Wani, M. C., Taylor, H. L., Wall, M. E., Coggon, P., \& McPhail, A. T. (1971). Journal of American Chemical Society, 93, 2325.

Yang, S.-J., Fang, J.-M., \& Cheng, Y.-S. (1996). Phytochemistry, 43, 839.

Yeh, M.-K., Wang, J.-S., \& Chen, F.-C. (1988). Journal of Chinese Chemical Society, 35, 309.

Zhang, S., Lee, C. T.-L., Chen, K., Kashiwada, Y., Zhang, D., McPhail, A. T., \& Lee, K.-H. (1994). Journal of Chemical Society, Chemical Communications, 1561. 